\title{
Activity statistics, avalanche kinetics, and velocity correlations in surface growth
}

\author{
Juan M. López* \\ Instituto de Física de Cantabria (IFCA), CSIC-UC, E-39005 Santander, Spain
}

Marc Pradas ${ }^{\dagger}$ and A. Hernández-Machado

Departament d'Estructura i Constituents de la Matèria, Universitat de Barcelona, Avinguda Diagonal 647, E-08028 Barcelona, Spain

(Received 3 September 2009; revised manuscript received 12 August 2010; published 22 September 2010)

\begin{abstract}
We investigate the complex spatiotemporal dynamics in avalanche driven surface growth by means of scaling theory. We study local activity statistics, avalanche kinetics, and temporal correlations in the global interface velocity, obtaining different scaling relationships among the involved critical exponents depending on how far from or close to a critical point the system is. Our scaling arguments are very general and connect local and global magnitudes through several scaling relationships. We expect our results to be applicable in a wide range of systems exhibiting interface kinetic roughening driven by avalanches of local activity, either critical or not. As an example we apply the scaling theory to analyze avalanches and roughening of forced-flow imbibition fronts in excellent agreement with phase-field numerical integrations.
\end{abstract}

DOI: 10.1103/PhysRevE.82.031127

PACS number(s): 05.40.-a, 47.56.+r, 64.60.Ht, 68.35.Ct

\section{INTRODUCTION}

The dynamics of rough surfaces in systems exhibiting avalanches or bursts of activity in response to a slow external driving has attracted a great deal of attention in recent years. Physical examples include fracture cracks [1], fluid imbibition fronts in porous media [2-4], or the motion of domain walls in magnets [5]. By means of high resolution fast imaging techniques it has recently been possible to characterize experimentally avalanche dynamics of activity from either the local velocity map [1] or the average velocity time series $[4,6]$. A fundamental problem is to understand the role played by local fluctuations (avalanches) in the building up of large-scale space-time interface correlations and scaleinvariant roughening.

In this paper, we show that local activity statistics, avalanche scaling properties, and time correlations (in both, the surface and the global velocity fluctuations) are generically connected through scaling relations that we derive by means of simple scaling arguments. Depending on the form of the probability distribution of the first-return time of activity to a given site, we divide avalanche driven surface growth systems into two separate classes as follows. We consider that driven systems can exhibit either off-critical or critical (fractal) spatiotemporal activity, and the scaling relations among the corresponding exponents are different for each class. On the one hand, there are systems where scale-invariant interface growth emerges from off-critical spatiotemporal patterns of activity, where avalanches have a finite extent and the probability for the activity to return to any given place is short tailed. On the other hand, there are growing interfaces, where local activity is distributed according to a fat-tailed

\footnotetext{
*1opez@ifca.unican.es

†Present address: Department of Chemical Engineering, Imperial College London, SW7 2AZ London, UK; m.pradasgene@imperial.ac.uk

†aurora@ecm.ub.es
}

power law, showing fractal patterns in space and/or time. In both cases, however, we find that the interface space-time correlations turn out to be related to the spatiotemporal activity, leading then to a connection between local and global quantities via scaling relationships-some of them different for off-critical and critical cases-that we obtain. Our arguments are very general and we expect our results to be widely applicable in systems where surface roughness fluctuations are driven by local avalanches. Examples where these ideas apply include systems near the depinning transition [7-15] as well as interface systems that self-organize into a critical state [16-24].

As an example of much current interest, we apply the scaling theory to the problem of forced-flow imbibition fronts (see Ref. [25] for a recent review), which occurs when a viscous fluid advances through a disordered media displacing a less viscous fluid (typically air). In forced-flow imbibition experiments a constant fluid injection rate is applied while the spatially averaged velocity of the liquid-air interface $\bar{v}$ is kept constant [4]. This system exhibits a natural characteristic length scale $\xi_{\times} \sim(1 / \bar{v})^{1 / 2}[25-29]$ corresponding to the typical extent of interface correlations, which can be controlled by the injection rate. Therefore, imbibition offers an excellent example where the theory we shall present here can be tested, since the system may be either in an off-critical regime $(\bar{v} \gg 0)$ with avalanches extending over short length scales of order $\xi_{\times}$, or fronts can be driven into a critical regime $(\bar{v} \rightarrow 0)$ by simply tuning the fluid injection rate [29]. Interestingly, these regimes can be accessible to experimentation [4].

The paper is organized as follows. In Sec. II, we study the local aspects of the interface dynamics in terms of activity statistics, where we give the exact definitions of critical and off-critical activity. We also study there the avalanche kinetics, where we present the different scaling relations among the local quantities describing avalanches. In Sec. III, we analyze the global dynamics of the interface by means of the multiscaling properties of the surface and the mean interface velocity time correlations. All the different quantities defined throughout the paper are then studied numerically in forced- 
flow imbibition fronts in Sec. IV. The final conclusions are given in Sec. V.

\section{LOCAL DYNAMICS}

\section{A. Activity statistics}

We consider an advancing interface $h(\mathbf{x}, t)$ in $d+1$ dimensions where $\mathbf{x}$ is the substrate position and $t$ is time. A site $\mathbf{x}$ is said to be active at a given time if it is moving. For discrete models this corresponds to sites to be updated, $h \rightarrow h$ +1 , in the next time step. For continuous systems defining single-site activity is more cumbersome but it can be effectively done by considering a site is active whenever the local velocity is above some fixed threshold. In order to characterize the local spatiotemporal activity we calculate the firstreturn time probability density, $\mathcal{P}_{\mathrm{f}}(T)$, for a site $\mathbf{x}$ to become active again after a period of inactivity $T$. This probability describes the time duration of intervals separating subsequent returns of activity at any given site so that the average number of returns $\mathcal{N}(T)$ in a time interval of duration $T$ satisfies [19]

$$
\mathcal{N}(T)=T-\mathcal{N}(T) \int_{\delta T}^{T} u \mathcal{P}_{\mathrm{f}}(u) d u
$$

where the temporal resolution can be set to unity, $\delta T=1$, without loss of generality.

In systems far from a critical point, which we denote as off-critical systems, activity is expected to be described by a general scaling function,

$$
\mathcal{P}_{\mathrm{f}}(T)=a\left(T_{0}\right) f\left(T / T_{0}\right),
$$

such that $f(y) \rightarrow 0$ as $y \rightarrow \infty$ and $a\left(T_{0}\right)$ is a normalization constant so that $\int^{\infty} d u \mathcal{P}_{\mathrm{f}}(u)=1$ is fulfilled. The average return time in this case is simply

$$
\langle T\rangle=\int_{1}^{\infty} u \mathcal{P}_{\mathrm{f}}(u) d u \propto T_{0},
$$

and thus a finite average surface velocity $\bar{v} \propto 1 /\langle T\rangle$ exists. A very common example are systems where the return of activity is a Poison process, so that the time interval distribution is simply exponential $f\left(T / T_{0}\right) \sim \exp \left(-T / T_{0}\right)$. In the case of systems exhibiting some degree of correlation between two consecutive returns of activity to a given site the distribution is more likely to be described by

$$
\mathcal{P}_{\mathrm{f}}(T) \sim T^{-\tau_{\mathrm{f}}^{\prime}} \exp \left(-T / T_{0}\right)
$$

where $\tau_{\mathrm{f}}^{\prime}$ represents an effective exponent characterizing the power-law regime. This case will also be classified as offcritical here since there still exists a finite average surface velocity with the average return time also given by Eq. (3).

In contrast, under certain circumstances, some systems may show patterns of activity recurrence that become critical or fractal (i.e., scale-invariant) in time in such a way that the first-return time probability density exhibits a power-law asymptotic tail,

$$
\mathcal{P}_{\mathrm{f}}(T) \sim T^{-\tau_{\mathrm{f}}} \quad \text { for } \quad T \gg 1,
$$

with exponent $\tau_{\mathrm{f}}>1$ for the distribution to be properly normalizable. In this case, in the long time limit we obtain that the average number of return points is $\mathcal{N}(T) \sim T^{\tau_{\mathrm{f}}-1}$ with some critical exponent within the range $\tau_{\mathrm{f}} \in(1,2]$. Note that, for $\tau_{\mathrm{f}}>2$, although the activity is still described by a power law, the system is actually off-critical due to the existence of a finite return time $\langle T\rangle$, i.e., returns become dense in time, and the interface advances at finite velocity $\left(\partial_{T} \mathcal{N} \sim\right.$ const. $)$. In contrast, for $1<\tau_{\mathrm{f}} \leq 2$ returns rarely occur and activity is said to be fractal in time. In this case, the average return time is infinite,

$$
\langle T\rangle \sim \int_{1}^{\infty} u^{1-\tau_{\mathrm{f}}} d u \rightarrow \infty,
$$

which is typical of a critical dynamics. Fractal activity occurs, for instance, near a pinning/depinning critical transition $(\bar{v} \rightarrow 0)$ or in surface growth models that self-organize into the critical state.

\section{B. Avalanche kinetics}

An avalanche is defined as a spatially connected cluster of active sites. Avalanches are characterized by the typical size $s(\ell)$ of an event of lateral spatial extent $\ell$. The duration and lateral extent of an event are related, $\ell \sim t^{1 / z_{\mathrm{av}}}$, via the avalanche dynamic exponent $z_{\mathrm{av}}$. One expects the average avalanche size to scale with the lateral extent up to the cutoff length scale (if any): $s(\ell) \sim \ell^{d_{\mathrm{av}}}$ for $\ell \ll \xi_{\times}$, where $d_{\mathrm{av}}$ is the avalanche dimension exponent. Conversely, one also expects to observe a scaling relation $s(t) \sim t^{\gamma}$ between size $s$ and duration $t$ of avalanches. Since an avalanche of lateral length $\ell$ leads to a fluctuation of the surface height of the order of the local width $w(l), d_{\mathrm{av}}$ can be easily related to the local roughness exponent $\alpha_{\text {loc }}$. Indeed, one has $s(\ell) \sim \ell^{d} w(\ell) \sim \ell^{d} \ell^{\alpha_{\mathrm{loc}}}$, and $d_{\mathrm{av}}=d+\alpha_{\mathrm{loc}}$ for a surface in $d+1$ dimensions. Likewise, the avalanche size-duration exponent $\gamma$ can then be obtained from the identity $s \sim t^{\gamma} \sim \ell^{d+\alpha_{\text {loc }}}$ and we find

$$
\gamma=\frac{\alpha_{\mathrm{loc}}+d}{z_{\mathrm{av}}},
$$

which connects avalanche dynamics with surface roughness [2].

In the case of off-critical activity, noncritical avalanches with a typical spatial extent cutoff $\xi_{\times}$are expected so that durations are also bounded and scaling only holds for $t$ $\ll t_{\times} \sim \xi_{\times}^{z a v}$. The avalanche size can be written in terms of the average velocity associated with the event as $s \sim \ell^{d} \mathcal{N}(t)$ $\sim \ell^{d} v(\ell) t$, for an avalanche of lateral extent $\ell$ and duration $t$. We assume that the typical velocity of the avalanche scales with the avalanche size as $v(\ell) \sim \ell^{-\delta}$. The identity $s$ $\sim \ell^{d} v(\ell) t \sim \ell^{d+\alpha_{\text {loc }}}$ leads to $\ell^{z_{\mathrm{av}}-\delta} \sim \ell^{\alpha_{\mathrm{loc}}}$ and we obtain the scaling relation

$$
z_{\mathrm{av}}=\alpha_{\mathrm{loc}}+\delta,
$$

relating surface roughness and avalanche exponents in the case of off-critical activity (noncritical avalanches). The ex- 
ponent $\delta$ in Eq. (8) gives account of possible interevent correlations. For instance, in the case of uncorrelated avalanches, typically, one should expect Gaussian fluctuations of the avalanche velocity and $\left\langle v(\ell) v\left(\ell^{\prime}\right)\right\rangle \sim \delta^{d}\left(\ell-\ell^{\prime}\right)$, giving $v(\ell) \sim \ell^{-d / 2}$. Avalanches are initiated in places favored given the disordered background. If this disorder is spatially uncorrelated it follows that avalanche velocity should also be uncorrelated and $\delta=d / 2$ [2]. Correlations in the random medium can produce non trivial correlations in the avalanche velocity and that possibility is contemplated here. Also, in the case of non disordered systems, where movement is driven by some external noise, the same argument applies since the noise may be either white or correlated. We have assumed a general situation by introducing the exponent $\delta$ that accounts for possible velocity correlations in case they exist.

A different result is obtained for driven surfaces exhibiting a fractal profile of activity which leads to critical avalanches with no characteristic length or time scales $\left(t_{\times} \rightarrow \infty\right)$. This is an interesting case because it includes surfaces near the pinning/depinning transition, surfaces driven into self-organized critical states, and surfaces obeying extremal dynamics, among other surface models in the critical state. As discussed above, avalanches in the presence of fractal activity do not have a finite velocity since the average number of returns of activity to a given site is $\mathcal{N}(t) \sim t^{\tau_{\mathrm{f}}-1}$ with $\tau_{\mathrm{f}}<2$. This means that the scaling relation (8) does not apply. However, the scaling relation between avalanche size and local width, $s \sim \ell^{d} \mathcal{N}(t) \sim \ell^{d} w(\ell)$, is indeed valid also in this case, but with $\mathcal{N}(t) \sim t^{\tau_{\mathrm{f}}-1}$. Thus we find the scaling relation [30]

$$
\tau_{\mathrm{f}}=\frac{\alpha_{\mathrm{loc}}}{z_{\mathrm{av}}}+1,
$$

which connects the local roughness with the statistics of the first-return time of surfaces exhibiting a fractal activity given by Eq. (5).

\section{GLOBAL DYNAMICS}

\section{A. Multiscaling of the height-height correlations}

Having activity in the form of localized avalanches implies that growth is highly inhomogeneous in space with many time scales involved, which, in turn, may produce multiscaling of the height-height correlations [18]. As it is shown below, this can give information about local properties through global observables. To this end we define $\Delta h\left(x, t ; t_{0}\right) \equiv h\left(x, t+t_{0}\right)-h\left(x, t_{0}\right)$ and investigate the generalized $q$-height-height correlation function in the saturated regime

$$
C_{q}(t) \equiv\left\langle\overline{\left|\Delta h\left(x, t ; t_{0}\right)-\overline{\Delta h\left(x, t ; t_{0}\right)}\right|^{q}} 1 / q\right\rangle \sim t^{\beta_{q}},
$$

where the over bar is a spatial average and brackets denote average over independent realizations. When the exponents $\beta_{q}$ depend on $q$ the surface is said to exhibit multiscaling and this indicates a highly nontrivial probability distribution of the height fluctuations. We are interested in obtaining $\beta_{q}$ as a function of the roughness and activity exponents.
In the limit $q \rightarrow \infty$ only the site with the maximum growth, $\Delta h_{\max }(t) \equiv\left\langle\max _{x}\left\{\Delta h\left(x, t ; t_{0}\right)\right\}\right\rangle$, contributes and we have $C_{\infty}(t) \approx \Delta h_{\max }(t)$. Note that $\Delta h_{\max }(t)$ becomes an important quantity since it corresponds to the typical height fluctuation produced on the surface by avalanche events in a time interval $t$. For time intervals $t$ shorter than the average avalanche duration cutoff $\left(t \ll t_{\times}\right)$we have $\Delta h_{\max }(t) \sim t^{\beta_{\infty}}$, where the exponent is $\beta_{\infty} \equiv \lim _{q \rightarrow \infty} \beta_{q}$. In contrast, in time scales longer than the typical event duration $\left(t \gg t_{\times}\right)$surface fluctuations are no longer spatially localized-very many avalanches occurring at different parts of the surface overlap and contribute to the fluctuation. Therefore surface height fluctuations must become spatially homogeneous at long time scales $\left(t \gg t_{\times}\right)$and the typical growth $\Delta h_{\max }(t)$ should scale with the global surface width $W(t) \sim t^{\alpha / z_{\text {av }}}$, where $\alpha$ is the global roughness exponent. These two limiting behaviors can be put as

$$
\Delta h_{\max }(t) \sim\left\{\begin{array}{ll}
t^{\beta_{\infty}} & \text { for } t \ll t_{\times} \\
t^{\alpha / z_{\mathrm{av}}} & \text { for } t \gg t_{\times}
\end{array},\right.
$$

where the crossover time $t_{\times}$is the average avalanche duration. In the case of off-critical activity $t_{\times}$is finite, while it diverges for fractal activity.

For a system of lateral extent $L$, surface fluctuations over time scales $t \ll t_{\times}$involve localized avalanche events of activity and so only a fraction of the surface $n_{\text {mov }} \sim(\ell / L)^{d}$ $\sim\left(t^{1 / z_{\mathrm{av}} / L}\right)^{d}$ is moving. We can then estimate the scaling of the term $\overline{(\Delta h)^{m}} \sim n_{\text {mov }}(t)\left[\Delta h_{\max }(t)\right]^{m} \sim\left(t^{1 / z_{\text {av }}} / L\right)^{d}\left[\Delta h_{\max }(t)\right]^{m}$ for $m>1$ and, using Eq. (11), we obtain

$$
\overline{(\Delta h)^{m}} \sim t^{m \beta_{\infty}+d / z_{\mathrm{av}}} L^{-d},
$$

for fluctuations at time scales $t \ll t_{\times}$, while it becomes

$$
\overline{(\Delta h)^{m}} \sim t^{m(2 \alpha+d) / 2 z_{\mathrm{av}}} L^{-m d / 2},
$$

for $t \gg t_{\times}$. Now, note that in the temporal regime $t \ll L^{z_{\mathrm{av}}}$ of interest here, the main contribution in Eq. (10) comes from the term $\overline{(\Delta h)^{q}} 1 / q$. Therefore we can write $C_{q}(t) \sim t^{\beta_{q}}$, with the $q$-dependent exponent

$$
\beta_{q}=\left\{\begin{array}{lll}
\beta_{\infty}+\frac{d}{q z_{\mathrm{av}}} & \text { for } t \ll t_{\times} \\
\frac{2 \alpha+d}{2 z_{\mathrm{av}}} & \text { for } t \gg t_{\times}
\end{array},\right.
$$

that gives the multiscaling exponents $\beta_{q}$ induced in the surface fluctuations by the localized avalanche dynamics, valid for both off-critical (finite $t_{\times}$) and fractal (unbounded $t_{\times}$) distributions of activity.

The exponent in the $q \rightarrow \infty$ limit appearing in Eqs. (14) and (11) is not an independent exponent and can be also obtained by a simple scaling argument in terms of the usual kinetic roughening exponents as follows. A height fluctuation at time scales $t \ll t_{\times}$corresponds to an avalanche of lateral extent $\ell$ and is given by $\Delta h_{\max } \sim s(t) / \ell^{d} \sim t^{\gamma-d / z_{\text {av }}}$. Using Eq. (7) we have $\beta_{\infty}=\alpha_{\text {loc }} / z_{\mathrm{av}}$. This means that the multiscaling exponents $\beta_{q}$ given by Eq. (14) are not independent indexes but can be expressed in terms of $\alpha_{\text {loc }}$ and $z_{\text {av }}$, the local roughness and dynamic exponent, respectively. 
The presence of multiscaling is due to the infinitely many time scales involved in the problem that are induced by a highly inhomogeneous surface growth. An illustrative way to show this is to note that the time scale defined by $C_{1}(t)$ $\sim t^{\beta_{1}}$ is associated with the avalanche size-duration exponent $\gamma$ since, from Eq. (14), $\beta_{1}=\beta_{\infty}+d / z_{\mathrm{av}}=\left(\alpha_{\mathrm{loc}}+d\right) / z_{\mathrm{av}}=\gamma[\mathrm{cf}$. Eq. (7)]. In the other limit, when $q \rightarrow \infty$, we have $\beta_{\infty}$ $=\alpha_{\mathrm{loc}} / z_{\mathrm{av}}$ that corresponds to the time scale of single-site activity. This observation is transparent for a fractal activity distribution where $\Delta h_{\max } \sim t^{\beta_{\infty}}$ can be related to the average number of returns to a given site, $\Delta h_{\max } \sim \mathcal{N}(t) \sim t^{\tau_{\mathrm{f}}-1}$. This gives $\tau_{\mathrm{f}}=\beta_{\infty}+1$, which corresponds to the previously derived scaling relation (9). This allows us to immediately associate the $\infty$-correlation moment (a global observable) with the distribution of the return times of activity at any given site (a local observable). This is useful for experiments, where single-site motion, and therefore local observables, may be difficult to measure.

\section{B. Extremal models of interface depinning}

It is interesting to particularize these results for the case of models exhibiting extremal dynamics [16,18,20-23] where only the most unstable site moves forward at each time step. These type of systems are right at the critical point by construction so that $t_{\times} \rightarrow \infty$ with no need of fine tuning any external field to bring the system into the critical state. The avalanche-duration exponent is $\gamma=1$ (only one site is growing at any given time) and we have $\Delta h_{\max }(t) \sim s(t) / \ell^{d}$ $\sim t / t^{d / z_{\mathrm{av}}}$ and $\beta_{\infty}=1-d / z_{\mathrm{av}}$, in agreement with existing results for critical depinning models with extremal dynamics [18]. As for the $\beta_{q}$ exponents they are predicted to be $\beta_{q}$ $=1+\left(d / z_{\mathrm{av}}\right)(1-q) / q$ by Eq. (14) after inserting $\beta_{\infty}=1$ $-d / z_{\mathrm{av}}$. In this case the model is at the critical state by construction so that $t_{\times} \rightarrow \infty$ and multiscaling takes place for the whole temporal range before saturation-the second branch given by Eq. (13) is never realized in this case.

Moreover, from Eq. (7) with $\gamma=1$ we obtain that the relation $z_{\mathrm{av}}=\alpha_{\mathrm{loc}}+d$ should be generically valid for extremal models of depinning in $d+1$ dimensions. This scaling relation is in agreement with existing numerical results $[22,23]$ (see also footnote [31]).

Our theory also gives correctly the growth exponent of the surface width in the intermediate regime before the stationary state. We have that the surface width at time $t$ is $W(t, L)=\left\langle\overline{\left[h\left(x, t+t_{0}\right)-h\left(x, t_{0}\right)\right]^{2}} 1 / 2\right\rangle=\left\langle\overline{(\Delta h)^{2}} 1 / 2\right\rangle$ and, using Eq. (12) with $m=2$ and inserting $z_{\mathrm{av}}=\alpha_{\mathrm{loc}}+d$, we obtain that the global width scales as $W \sim t^{\beta} L^{-d / 2}$ with $\beta=\left(\alpha_{\text {loc }}+d / 2\right) /\left(\alpha_{\text {loc }}\right.$ $+d)$, in agreement with existing results for depinning models with extremal dynamics [14,22-24] (see also Ref. [31]). Note that we obtain in a very natural way the residual dependence $L^{-d / 2}$ that has been found to be generic for models of interface depinning [18,22-24].

\section{Correlations of the global velocity fluctuations}

Fluctuations of the average surface velocity are expected when a system is externally driven even if the driving force is time independent. Recently, there has been much interest
$[2,4,6,32,33]$ in obtaining information about the avalanche kinetics from the scaling behavior of the global activity/ velocity fluctuations. This is particularly important in experiments where the global velocity time series is readily available, while it is difficult to monitor microscopic avalanches. The scaling relations obtained in the preceding sections can be used to give a full solution to this problem in the context of surface growth.

In the stationary state, $t_{0} \gg L^{z_{\mathrm{av}}}$, we consider the velocityvelocity correlation $\Delta v(t)=\left\langle\left[v\left(t+t_{0}\right)-v\left(t_{0}\right)\right]^{2}\right\rangle$, where $v(t)$ is the average instantaneous velocity time series. Equivalently, we can compute the velocity-fluctuation spectrum $\left\langle|\hat{v}(\omega)|^{2}\right\rangle$, where $\hat{v}(\omega)$ is the Fourier component at frequency $\omega \sim t^{-1}$. Generally, one expects $\Delta v(t) \sim t^{2 H}$ where $H$ is Hurst exponent of the velocity time series, while in Fourier space we have $\left\langle|\hat{v}(\omega)|^{2}\right\rangle \sim \omega^{-\nu}$, with $\nu=2 H+1$. Since $v(t) \equiv \partial_{t} \bar{h}$, the velocity-fluctuation spectrum can be written as $\left\langle|\hat{v}(\omega)|^{2}\right\rangle$ $=\omega^{2}\left\langle|\hat{\bar{h}}(\omega)|^{2}\right\rangle$. We are interested here in the case of surfaces exhibiting kinetic surface roughening in $d+1$ dimensions where the structure factor describing the height-height correlations scales as

$$
\left\langle|\hat{h}(\mathbf{k}, t)|^{2}\right\rangle=k^{-(2 \alpha+d)} f\left(k t^{1 / z_{\mathrm{av}}}\right),
$$

where $k=|\mathbf{k}|$, and the scaling function $f(u) \sim u^{2 \alpha+d}$ for $u$ $\rightarrow 0$. We have

$$
\left\langle|\hat{\bar{h}}(\omega)|^{2}\right\rangle=\lim _{\mathbf{k} \rightarrow 0}\left\langle|\hat{h}(\mathbf{k}, \omega)|^{2}\right\rangle \sim(1 / \omega)^{(2 \alpha+d) / z_{\mathrm{av}}+1},
$$

leading to the scaling exponent

$$
\nu=\frac{2 \alpha+d}{z_{\mathrm{av}}}-1,
$$

which relates the low-frequency behavior of the velocityfluctuation spectrum to the roughness properties of the surface. This scaling behavior holds for frequencies above $\omega_{0}$ $\sim(1 / L)^{z_{\text {av }}}$, below which the spectrum becomes flat. This formula generalizes an earlier analytical result [34], $\nu=(d+4) / z-3$, valid for the Kardar-Parisi-Zhang equation [35].

\section{CASE STUDY: FLUID IMBIBITION IN RANDOM MEDIA}

We now apply the above scaling theory to the problem of forced-flow imbibition in disordered media [25]. As it has been noted in the introduction, the main point in forced-flow imbibition lays in the existence of a natural characteristic length $\xi_{\times} \sim(1 / \bar{v})^{1 / 2}$ for the typical avalanche extent [25-29], which can be controlled by the liquid flow rate. In this way, we have a system whose activity can be made progressively fractal as the velocity is tuned from moderate to very low values.

We simulate forced-flow imbibition in $1+1$ dimensions in the capillary dominated regime by integrating a standard phase-field model [25-29] 

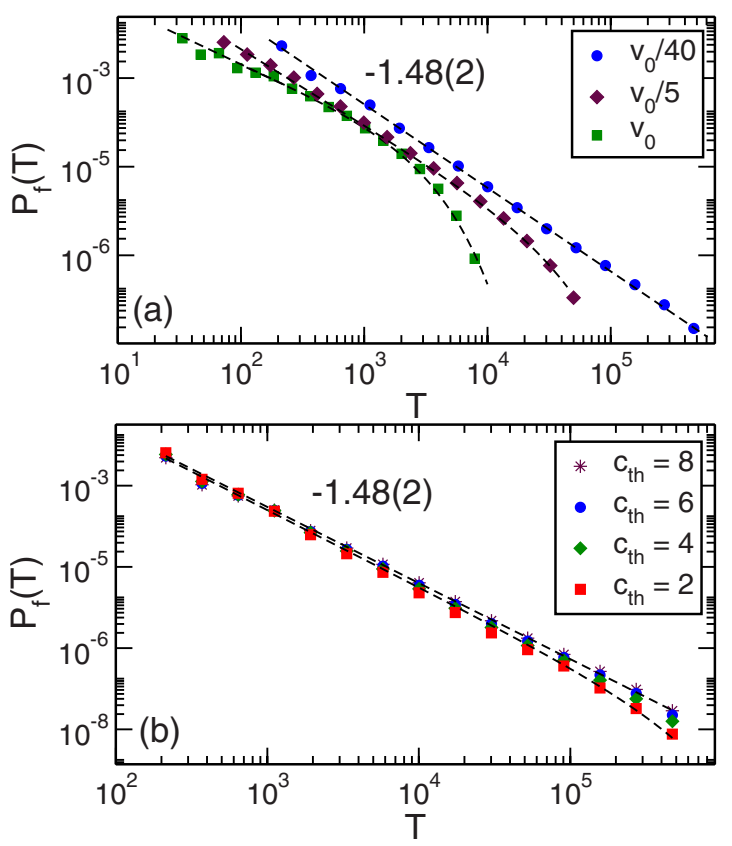

FIG. 1. (Color online) (a) First-return time probability density $P_{\mathrm{f}}(T)$ calculated at different velocities. It reflects an off-critical activity (moderate velocities: $\bar{v}=v_{0}$ and $\bar{v}=v_{0} / 5$ ), characterized by: $\mathcal{P}_{\mathrm{f}}(T) \sim T^{-\tau_{\mathrm{f}}^{\prime}} \exp \left(-T / T_{0}\right)$ with effective exponents of $\tau_{\mathrm{f}}^{\prime} \simeq 1.0$ and 1.37 , respectively. Fractal activity is found only for low enough velocities. In the panel we plot the distribution for $v=v_{0} / 40$ where $\mathcal{P}_{\mathrm{f}}(T) \sim T^{-\tau_{\mathrm{f}}}$ with $\tau_{\mathrm{f}}=1.48 \pm 0.02$. All results shown correspond to a threshold value of $c_{\mathrm{th}}=6$. (b) Effect of the choice of a velocity threshold $c_{\mathrm{th}}$. First-return time probability density for the case of $\bar{v}=v_{0} / 40$, when the single-site activity is critical, calculated by choosing different values of the threshold $c_{\mathrm{th}}$. The distribution can be fitted by $\mathcal{P}_{\mathrm{f}}(T) \sim T^{-\tau_{\mathrm{f}}} \exp \left(-T / T_{\mathrm{th}}\right)$ with $T_{\mathrm{th}}$ depending on $c_{\mathrm{th}}$. Note that $\tau_{\mathrm{f}} \simeq 1.48$ is robust for different velocity thresholds $c_{\mathrm{th}}$ indicating it is a critical exponent.

$$
\partial_{t} \phi=\nabla M \nabla\left[-\phi+\phi^{3}-\epsilon^{2} \nabla^{2} \phi-\eta(\boldsymbol{r})\right],
$$

in a two-dimensional system of lateral size $L=512$ with $\epsilon=1$. The quenched random field $\eta(\boldsymbol{r})>0$ models capillary disorder and favors the liquid (wet) phase, forcing the interface to advance at the expense of the air (dry) phase. The parameter $M$ in the above equation is the mobility which we take constant at the liquid phase $(\phi>0)$ and zero at the air phase $(\phi<0)$. In our numerical model we have used a spatially distributed dichotomic quenched noise and the results have been averaged over 25 disorder realizations. The interface position $h(x, t)$ separating the wet and dry phases is computed. A site on the interface is active at time $t$ if its local velocity is above some fixed threshold, $v(x, t)>c_{\mathrm{th}} \bar{v}$, where $c_{\text {th }}$ is some arbitrary constant. In the case of critical activity, exponents should not depend on $c_{\text {th }}$, which only determines a lower cutoff velocity to define a site advance.

In Fig. 1(a) we plot the distribution of first-return times of activity to any given site. We see that for moderate velocities, $\bar{v}=v_{0}=2 \times 10^{-3}$ and $\bar{v}=v_{0} / 5$, the activity is an exponentially cutoff power law with an exponent that depends on the mean velocity, while it tends to be pure power-law dis-
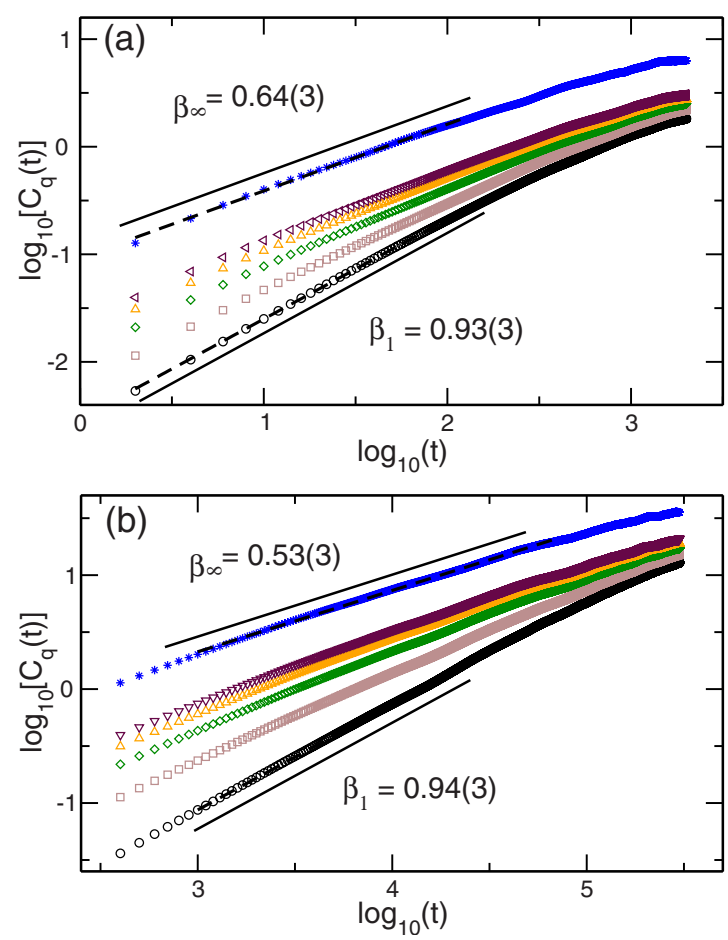

FIG. 2. (Color online) Height-height time correlation function $C_{q}(t) \sim t^{\beta_{q}}$, calculated at two typical velocities, shows the presence of multiscaling with $\beta_{1} \approx 0.93, \beta_{2} \approx 0.81, \beta_{3} \approx 0.73, \beta_{4} \approx 0.70$, and $\beta_{\infty} \approx 0.64$ at moderate velocities, $\bar{v}=v_{0}(\mathrm{a})$, and $\beta_{1} \approx 0.94, \beta_{2}$ $\approx 0.75, \beta_{3}=0.67, \beta_{4} \approx 0.63$, and $\beta_{\infty} \approx 0.53$ at low velocities, $\bar{v}=v_{0} / 40(\mathrm{~b})$.

tributed for very low velocities, $\bar{v}=v_{0} / 40$, where we obtain $\tau_{\mathrm{f}}=1.48 \pm 0.02$ over a range of more than three decades. These distributions of activity correspond to the fact that the characteristic avalanche size (and duration) diverges as one gets closer to the pinned state, $\bar{v} \rightarrow 0$ [29]. It is worth to mention here that the observed results do not depend on the choice of the arbitrary threshold $c_{\text {th }}$. Figure 1(b) shows that changing the value of $c_{\text {th }}$ only introduces an artificial cutoff but does not change the exponent, something already observed in Ref. [29].

Let us now study these two different dynamical regimes. In the case of moderate velocities (off-critical activity) the stationary surface is super-rough [36] and recent numerical work shows $[2,29]$ that the global and local roughness exponents are, $\alpha \approx 5 / 4$ and $\alpha_{\text {loc }}=1$, respectively. Avalanches in imbibition are driven by the capillary disorder [2], which is a random quenched and uncorrelated field, so we should expect the Gaussian value $\delta=1 / 2$. Inserting these values in Eqs. (7) and (8), we find the dynamic exponent $z_{\mathrm{av}}=3 / 2$ and the avalanche-duration exponent $\gamma=4 / 3$ in $d=1$, in excellent agreement with the existing numerical estimates [2,29] for imbibition fronts in this range of velocities. Figure 2 shows that the surface fluctuations display multiscaling up to a given characteristic time scale. In particular, we predict $\beta_{\infty}$ $=\alpha_{\mathrm{loc}} / z_{\mathrm{av}}=2 / 3$, which is in excellent agreement with the numerical estimate in Fig. 2(a). However, Eq. (14) with $\alpha_{\mathrm{loc}}$ $=1$ and $z_{\mathrm{av}}=3 / 2$ only reproduces the trend of the numerical estimated exponents but not the exact values, as a clear consequence of the strong crossover effects due to the finite 




FIG. 3. (Color online) Power spectrum of the mean interface velocity $\left\langle|\hat{v}(\omega)|^{2}\right\rangle \sim \omega^{-\nu}$ calculated at two typical velocities, obtaining $\nu=1.26(8)$ at high velocities and $\nu=1.05(7)$ at low velocities in agreement with the theoretical prediction 1.33 and 1.0, respectively.

cutoff time scale $t_{\times}$. We expect our prediction for $\beta_{q}$ to become better as $t_{\times}$becomes larger (see below).

Regarding the velocity fluctuations spectrum we predict a decay as $\sim(1 / \omega)^{\nu}$ with $\nu$ given by Eq. (17) up to a cutoff frequency $\omega_{0} \sim\left(1 / \xi_{\times}\right)^{z_{a v}}$ associated with the saturation time in a finite-length correlated system. Replacing the known numerical estimates [2,29] for the critical exponents $\alpha=5 / 4$ and $z_{\mathrm{av}}=3 / 2$, we predict a velocity correlation exponent $\nu$ $=4 / 3$ in good agreement with our simulations $\nu$ $=1.26 \pm 0.08$ in Fig. 3 , as well as the numerical estimate in Ref. [2].

The scaling behavior changes dramatically when we drive the front at very low velocities, where the activity statistics becomes approximatively fractal (see Fig. 1). Although, for an infinite size system, a truly fractal activity distribution would only appear in the limit $\bar{v} \rightarrow 0$, we observe that for velocities as low as $\bar{v}=v_{0} / 40$ our finite system has a robust scale-invariant activity distribution $\mathcal{P}_{\mathrm{f}}(T) \sim T^{-\tau_{\mathrm{f}}}$ over four decades in $T$. We recall that, according to the arguments discussed in the previous sections, the scaling relation (8) is no longer valid for fractal activity and has to be replaced by Eq. (9). The scaling exponents $\alpha=3 / 2, \alpha_{\mathrm{loc}}=1, z_{\mathrm{av}}=2$, and $\gamma=1$ in this close-to-pinning regime have been recently obtained [29] both analytically and numerically by using the phasefield model described above. We are therefore able to check our scaling relations (9), (14), and (17). Replacing $\alpha_{\mathrm{loc}}=1$ and $z_{\mathrm{av}}=2$ in Eq. (9) we find the first-return time distribution exponent $\tau_{\mathrm{f}}=3 / 2$, which is in excellent agreement with our numerical estimate in Fig. 1.

Figure 2(b) shows that the system also exhibits multiscaling in the regime of low velocities. In this regime the system is very close to the pinning critical point (lacking characteristic lengths or time scales) and our prediction for the multiscaling exponents $\beta_{q}$ is expected to be more accurate. In fact, inserting $\alpha_{\mathrm{loc}}=1$ and $z_{\mathrm{av}}=2$ in Eq. (14) we obtain $\beta_{q}=(q+1) / 2 q$, which leads to the set of theoretical exponents $\beta_{1}=1, \beta_{2}=0.75, \beta_{3}=0.667$, and $\beta_{4}=0.625$ in excellent agreement with our simulations [cf. Fig. 2(b)]. Also, in the limit $q \rightarrow \infty$ we expect to have $\beta_{\infty}=\alpha_{\mathrm{loc}} / z_{\mathrm{av}}=1 / 2$ to be compared with the numerical result $\beta_{\infty} \approx 0.53$.

Finally, regarding the temporal correlations of the global velocity signal in the low-velocity regime we can replace $\alpha=3 / 2$ and $z_{\mathrm{av}}=2$ in Eq. (17) to obtain $\nu=1$ for the full range of frequencies, which is to be compared with our numerical estimate $\nu=1.05 \pm 0.07$ shown in Fig. 3 .

\section{CONCLUSIONS}

To summarize, we have presented a scaling theory of surface roughening in systems driven by avalanches exhibiting either off-critical activity distribution, described by a power law with a cutoff function, or critical activity distribution, characterized by a pure power law. Our results connect, on the one hand, distributions of local activity with the scaling properties of avalanches and surface roughness. On the other hand, temporal correlations in both the surface and the global velocity signal with local activity and avalanche kinetics also turn out to be connected by means of scaling relations.

In order to achieve the critical state two different driving mechanisms are typically used. On the one hand, one can get to the critical point by tuning the external driving force to the critical threshold [7-15]. In this case the system will exhibit fluctuations of the global velocity. On the other hand, one can drive the system so that the velocity is time independent (no fluctuating) but then the external force changes in time to keep the system right at the critical point. The latter can be achieved by extremal dynamics [16-24] or other prescriptions for self-organized criticality $[21,37]$. Throughout this paper, the most common case of a constant external driving force was assumed but our scaling theory can actually be applied to either way of driving. We have also shown how our results can be particularized to extremal models, where there are no fluctuations of the surface velocity.

Our arguments are very general and should be valid in very different contexts where surface roughness fluctuations are driven by local avalanches of motion, characterized by having either a parallel or extremal dynamics. For illustration, we have applied the theory to the problem of forcedfluid imbibition, which can show both off-critical and critical distributions of local activity as the average front velocity is tuned toward zero, obtaining in both cases excellent agreement between theory and numerical results. Our predictions for the single-site activity statistics, avalanche distribution, roughness, and velocity-fluctuation correlations are of interest for the experiments in this little explored region in the context of forced-imbibition near the depinning transition.

\section{ACKNOWLEDGMENTS}

This work was supported by the DGI (Ministerio de Educación y Ciencia, Spain) through Grant Nos. FIS200912964-C05-02 and 05. 
[1] K. J. Måløy, S. Santucci, J. Schmittbuhl, and R. Toussaint, Phys. Rev. Lett. 96, 045501 (2006).

[2] M. Rost, L. Laurson, M. Dubé, and M. J. Alava, Phys. Rev. Lett. 98, 054502 (2007).

[3] J. Soriano, A. Mercier, R. Planet, A. Hernández-Machado, M. A. Rodríguez, and J. Ortín, Phys. Rev. Lett. 95, 104501 (2005).

[4] R. Planet, S. Santucci, and J. Ortín, Phys. Rev. Lett. 102, 094502 (2009).

[5] J. P. Sethna, K. Dahmen, and C. Myers, Nature (London) 410, 242 (2001).

[6] S. T. Bramwell, Nat. Phys. 5, 444 (2009).

[7] D. S. Fisher, Phys. Rev. Lett. 50, 1486 (1983); Phys. Rev. B 31, 1396 (1985).

[8] O. Narayan and D. S. Fisher, Phys. Rev. Lett. 68, 3615 (1992); Phys. Rev. B 46, 11520 (1992); 48, 7030 (1993).

[9] T. Nattermann, S. Stepanow, L.-H. Tang, and H. Leschhorn, J. Phys. II 2, 1483 (1992).

[10] L.-H. Tang and H. Leschhorn, Phys. Rev. A 45, R8309 (1992).

[11] S. V. Buldyrev, A.-L. Barabasi, F. Caserta, S. Havlin, H. E. Stanley, and T. Vicsek, Phys. Rev. A 45, R8313 (1992).

[12] H. Leschhorn, Physica A 195, 324 (1993).

[13] Luis A. Nunes Amaral, A.-L. Barabasi, and H. E. Stanley, Phys. Rev. Lett. 73, 62 (1994).

[14] H. A. Makse and L. A. Nunes Amaral, EPL 31, 379 (1995).

[15] L.-H. Tang, M. Kardar, and D. Dhar, Phys. Rev. Lett. 74, 920 (1995).

[16] K. Sneppen, Phys. Rev. Lett. 69, 3539 (1992).

[17] K. Sneppen and M. H. Jensen, Phys. Rev. Lett. 71, 101 (1993).

[18] H. Leschhorn and L.-H. Tang, Phys. Rev. E 49, 1238 (1994).

[19] S. Maslov, M. Paczuski, and P. Bak, Phys. Rev. Lett. 73, 2162 (1994).

[20] Z. Olami, I. Procaccia, and R. Zeitak, Phys. Rev. E 49, 1232 (1994).

[21] M. Paczuski, S. Maslov, and P. Bak, Phys. Rev. E 53, 414 (1996).

[22] A. Tanguy, M. Gounelle, and S. Roux, Phys. Rev. E 58, 1577 (1998).

[23] S. Krishnamurthy, A. Tanguy, and S. Roux, Eur. Phys. J. B 15, 149 (2000).

[24] O. Narayan, Phys. Rev. E 62, R7563 (2000).
[25] M. Alava, M. Dubé, and M. Rost, Adv. Phys. 53, 83 (2004).

[26] M. Dubé, M. Rost, K. R. Elder, M. Alava, S. Majaniemi, and T. Ala-Nissila, Phys. Rev. Lett. 83, 1628 (1999).

[27] T. Laurila, C. Tong, I. Huopaniemi, S. Majaniemi, and T. AlaNissila, Eur. Phys. J. B 46, 553 (2005).

[28] M. Pradas and A. Hernández-Machado, Phys. Rev. E 74, 041608 (2006).

[29] M. Pradas, J. M. López, and A. Hernández-Machado, Phys. Rev. E 80, 050101(R) (2009).

[30] L. Laurson and M. J. Alava, Eur. Phys. J. B 42, 407 (2004).

[31] Notice that our scaling relations differ from those in Refs. [22-24] in that the local roughness exponent $\alpha_{\text {loc }}$ replaces the global one $\alpha$. In those works it is assumed that an avalanche event of extent $\ell$ has a size $W(\ell) \ell \sim \ell^{\alpha+1}$, where $W$ is the typical amplitude of a surface fluctuation. However, this is generally incorrect, in particular in the frequent situations where anomalous scaling [36] occurs and $\alpha \neq \alpha_{\text {loc. }}$. This has added much confusion when comparing with numerical results in cases where super-roughening $\left(\alpha>1\right.$ and $\left.\alpha_{\mathrm{loc}}=1\right)$ takes place (see for instance footnote [20] in Ref. [24] and references therein). We have instead considered here that the size of a single event is $w(\ell) \ell \sim \ell^{\alpha_{\mathrm{loc}}+1}$ where $w(\ell)$ is the local roughness. Individual avalanche events scale with the local roughness, $\alpha_{\text {loc }}$, while the global roughness exponent $\alpha$ describes the large scale surface fluctuations where one has contributions from many overlapping avalanches. Our scaling relations are correct in all the situations whether standard $\left(\alpha=\alpha_{\text {loc }}\right)$, superroughening $\left(\alpha>1\right.$ and $\left.\alpha_{\mathrm{loc}}=1\right)$ or intrinsic anomalous $(\alpha$ $\left.\neq \alpha_{\text {loc }}\right)$ scaling occur [36].

[32] M. C. Kuntz and J. P. Sethna, Phys. Rev. B 62, 11699 (2000)

[33] L. Laurson, M. J. Alava, and S. Zapperi, J. Stat. Mech.: Theory Exp. (2005) L11001; L. Laurson, X. Illa, and M. J. Alava, ibid. (2009) P01019.

[34] J. Krug, Phys. Rev. A 44, R801 (1991).

[35] M. Kardar, G. Parisi, and Y.-C. Zhang, Phys. Rev. Lett. 56, 889 (1986).

[36] J. J. Ramasco, J. M. López, and M. A. Rodríguez, Phys. Rev. Lett. 84, 2199 (2000).

[37] P. Bak, C. Tang, and K. Wiesenfeld, Phys. Rev. Lett. 59, 381 (1987). 